\title{
MENDELIAN VARIATION IN THE PARACOLON MUTABILE COLON GROUP AND THE APPLICATION OF MENDEL'S PRINCIPLES TO THE THEORY OF ACQUIRED VIRULENCE.
}

\author{
By F. H. STEWART, M.A., D.Sc., M.D., D.P.M., Major, I.M.S. (Ret.). \\ (Assistant Medical Officer, County Mental Hospital, Cheddleton.) \\ CONTENTS.

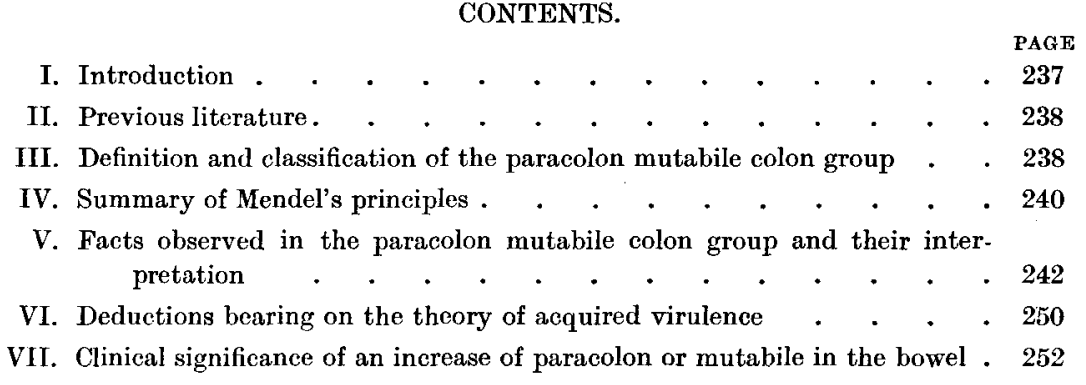

\section{Introduction.}

IT is the thesis of this paper that Mendel's principles of variation, formed from the study of the genetics of higher plants and animals, can be applied also to bacteria, and that acquired virulence in bacteria is the result of Mendelian variation.

This thesis is not entirely new. Neisser (1906) and Massini (1907) suggested that the variation observed by them in Bacterium coli mutabile was a mutation in the sense of De Vries, and a considerable number of papers have been published in support of or in opposition to this view. Toeniessen (1921), as the result of work on Bacillus pneumoniae Friedländer, claims that bacteria possess the same forms of variation as metazoa with the exception of that achieved through crossing. He distinguishes the following modes of variation in bacteria:

(1) Modification, change in response to external influence, which reverts when the external influence ceases to act.

(2) Alternation, due to change in dominance of unit characters (Valenzwechsel).

(3) Mutation, by loss or interpolation of unit characters.

He asserts that virulence varies through mutation.

Mendel's form of variation arising from (1) the segregation of existing unit characters in gametic division, and (2) their recombination by conjugation, is thus excluded by Neisser, Massini, and Toeniessen from the life processes of 
bacteria. This form of variation is, of course, the most frequent and important cause of change in higher plants and animals. I hope to be able to show that part (1) at least of this process operates constantly in bacteria, and that the phenomenon of acquired virulence is probably dependent on it.

This paper is based on a laboratory and clinical study of the group of intestinal bacteria known as paracolon mutabile colon. In order to place clearly before the reader the parallelism between the phenomena in higher plants and animals on the one hand, and the bacteria on the other, I will divide the main part of the paper into (1) a brief summary of Mendel's principles, divided into a series of subsections, with illustrative examples from higher forms, and (2) a description of the facts observed in the paracolon mutabile colon group, with the application of Mendel's principles to them, following the subsections distinguished above.

\section{Previous literature.}

The paracolon bacilli were first described by Gilbert and Lion (1893); Morgan (1906 and 1907), and Morgan and Ledingham (1909) classified them in their groups X, XII and XIV of non-lactose fermenters from the faeces. Mackie (1913), György (1921), Herrold and Culver (1919) studied them from the urine in pyuria and bacilluria, and described their serological characters.

Bacterium coli mutabile was first described by Neisser (1906) as the result of work carried out by Massini under his direction. Massini published an account in 1907. Since then the group of variable coliforms has been studied by Burck (1908), Reiner Müller (1909 and 1911), Burri and Düggeli (1909), Burri (1910), and Baerthlein (1911, 1912, 1918).

Penfold's work on variation in B. typhosus and B. coli (1911), and Henderson Smith's article (1913) on the differentiation of the coli-typhoid group, also contribute to the subject. Dobell reviewed variation in bacteria in 1913 .

Neisser and Massini regarded the variation from the non-lactose fermenting to the lactose fermenting condition as a mutation in the sense of De Vries. Burri (1910), on the other hand, contended that the change was not sudden and abrupt, but gradual, and therefore differed from mutation. His argument can be reconciled with Mendelian variation, the transitional forms being due to different degrees of dominance.

The allied subject of the "rough" variation in B. dysenteriae Shiga, and the Salmonella group, was introduced by Arkwright (1921), and was further studied by Schütze (1922), and by Arkwright and Goyle (1924). A full summary and discussion is contained in Savage and Bruce (1925).

\section{DeFinition AND Classification of the PARACOLON MUTABILE COLON GROUP.}

The group paracolon mutabile colon consists of gram-negative cocco-bacilli and bacilli, obtained chiefly from the alimentary canal, which do not liquefy gelatine, which give acid and gas in glucose, maltose, and mannite, and which 
are extremely individual in their antigenic power, an anti-serum to one strain reacting only to its homologous organism and its immediate derivatives. None of them is agglutinated by standard sera against the typhoid-paratyphoid group (Mackie, 1913; Herrold and Culver, 1919; György, 1921). Colon can regularly be derived from mutabile, and under special circumstances both mutabile and colon can be obtained from paracolon. It is probable that paracolon is also derived from mutabile1. I hope in this paper to show that the three subgroups are Mendelian variants of one species, mutabile being the central heterozygote.

All the members of the group when grown on solid culture media form colonies which bear papillae on their surface. The papillae are minute hemispherical projections varying from 0.05 to $1 \mathrm{~mm}$. in diameter. With one exception it is from these papillae that the known variations arise. Variation occurs in many characters, of which four have been studied in this laboratory: (1) sugar reactions, (2) capsulation of the bacteria accompanied by gelatinous transformation of the colony, (3) pattern and colour of the colony, (4) opacity and smoothness of the colony contrasted with translucency and roughness. The fourth character is that in which variation arises not in the papillae but in the body of the colony.

The table exhibits the classification of the various strains studied in the group paracolon mutabile colon. Subgroup I, paracolon, does not show routine variation in reaction to any sugar and does not ferment lactose. Subgroup II, mutabile, shows routine Mendelian variation to either lactose, saccharose, or dulcite, or to more than one. As originally defined by Neisser (1906) and Massini (1907), mutabile (Bact. coli mutabile) consisted of forms variable to lactose only, but there is no reason for restricting the term in this way, and I have removed those variable to saccharose and dulcite from the subgroup paracolon and included them here. B. imperfectum of Burri and Düggeli (1909) is a mutabile which varies to saccharose. Variable lactose fermenters have also been removed from the third subgroup, colon, and included under mutabile.

The races studied in this laboratory are distinguished by initials and numbers. Parent races as isolated from the bowel are those not marked "subrace." The "subraces" are the variants derived in culture from the native forms. The classification applies to the parent forms only, thus a number of lactose fermenting subraces will be found under paracolon, because they were derived from paracolon parents. The variants arise in two modes: (1) by rare variation or mutation, in which case the parent form is marked in the column "Mutation," with " $L$ " where the change is to lactose, and with " $\mathrm{S}$ " where it is to saccharose, with " $\mathrm{D}$ " where it is to dulcite, and with " $\mathrm{C}$ "

1 Henderson Smith (1913). "Penfold has brought forward evidence that parallel to the main group of lactose fermenters in the intestine, there are corresponding groups of non-lactose fermenters belonging to the mutabile group, and which are convertible into lactose fermenters. One group may arise from its correspondent under natural conditions." 
where it implies capsulation. (2) By routine (Mendelian) variation; races which form Mendelian variants are similarly marked in the column "Mendelian variation."

In the column "Capsule" " $\mathrm{L}$ " indicates that the strain capsulates on a lactose medium, "S" on saccharose, "G" on glucose, "D" on dulcite.

Taking the races seriatim as given in the table, and confining our attention to those which vary in sugar reactions, I (2) F.M.G. 512 produced by mutation the subrace 2638, 2638 by Mendelian variation gave 2473 and 2817 , and 2473 similarly gave 2169 , which by mutation gave 3353 , which by Mendelian variation gave 3351 and 3196. My reasons for assigning certain instances of change to mutation and others to Mendelian variation will be found in Sect. v, subsections A 2 and A 1.

Subgroup II: in all the races (with the exception of F.S. 3674 and T.A.B. 1678) the derived forms arise by Mendelian variation from the parents. In II D. (3) 1899 and 1888 arose from J.R. 506, and 1887 from 1899.

In II B. (1) race F.S. 3674 gave by Mendelian variation subrace 1900, and by mutation subrace 3305 , which latter by Mendelian variation gave 3329 .

In II C. (1) T.A.B. 1678 gave by mutation subrace 3453 , which differed from its parent in regularly forming red papillae on lactose, from which the subrace 3555 arose by Mendelian variation.

In the column "Coagglutination" those forms which are serologically identified are bracketed together. I may add that paracolon, mutabile, and colon are as a rule remarkably individual in their antigenic power. An antiserum against one race agglutinates that race and its immediate descendant subraces only. Similar races from other hosts are either not agglutinated or only in very low dilution. Although this is the rule, I was fortunate in securing one race of $B$. acidi lactici Hüppe, which was more catholic in antigenic power. An antiserum against this race, of titre 7500, agglutinated a derived $B$. $c$. communior (512. S.R. 2473), and a derived mutabile (S.R. 2638) to $1 / 2000$, and a mutabile (F.S. 249) to $1 / 5000$, while it did not agglutinate a pure paracolon (512 parent), or a derived Friedländer (512 S.R. 2618). This experiment indicates a structural affinity between colon and mutabile regardless of ancestry, and dissimilarity between naked colon on the one hand, and paracolon and Friedländer (or capsulated colon) on the other.

\section{Summary of Mendel's princtples.}

In order to marshal the evidence logically and to define the sense in which certain terms are used in this paper, it will be well to summarise Mendel's principles, and in the next section to apply them seriatim to the facts observed in the bacteria under consideration.

A. Variation occurs in two modes. (1) True Mendelian variation from the segregation of previously existing unit characters. Segregation occurs at every gametic division, but variation appears only if the parents are heterozygous or hybrid. For example, a hybrid between the tall and short races of pea is 
heterozygous for the tall unit character, and has a formula Tt. Tt mated with Tt gives descendants as follows: $1 \mathrm{TT}, 2 \mathrm{Tt}, 1 \mathrm{tt}$, i.e. one pure tall, two heterozygous talls, and one short. The heterozygous form continues to throw talls and shorts indefinitely, while the two pure forms breed true. (Bateson, 1913; Punnett, 1919.)

(2) The rarely occurring variation or mutation of De Vries due to:

(a) The dropping out of a pre-existing factor through some disturbance of cell division at the formation of the gametes. (Punnett, 1919, pp. 77, 78.) An imaginary example would be if in a homozygous tall pea the factor for tallness were to drop out, the homozygous tall TT thus giving rise to descendants lacking the tall factor and having a formula tt. Mutation has taken place from the tall to the dwarf habit.

(b) Similarly mutation may take place by the interpolation of a new factor, for example, if a dwarf pea, tt, lacking the tall factor, should by some disturbance in the gametic cell divisions give rise to descendants containing it, i.e. with a formula TT, mutation would have taken place by interpolation from the dwarf to the tall habit.

Mutation of type (a), though it does not recur regularly, is not uncommon; the origin of many of the cultivated varieties of plants, e.g. of the sweet pea, is due to a repeated dropping out of factors which were all present in the wild parent, as each factor dropped out in succession a new variety arose.

Mutation of type $(b)$ is, of course, less common than type $(a)$.

B. Dominance. Of two allelomorphs, e.g. T and $\mathrm{t}$ in a heterozygote, one may be dominant over the other, and in this case the heterozygote shows the dominant character in as full development as if it were homozygous for that character. Thus in a pea Tt, if $T$ is completely dominant to $t$, the plant is as tall as if its composition were TT. On the other hand, dominance may be incomplete or absent, and the heterozygote is intermediate in that character between the pure dominant $\mathrm{TT}$ and the pure recessive tt.

The presence and absence hypothesis assumes that the recessive character unit is merely the absence of the dominant, and that $t$, therefore, does not represent a positive character for the dwarf habit but merely the absence of the tall factor $\mathrm{T}$.

C. Inhibitory factors. A unit character may manifest its presence not in a positive manner, but by inhibition of another unit character present. Thus some races of rabbits and poultry are white, not from lack of a colour factor $\mathrm{C}$, but from its inhibition by an inhibitory factor I. They have the formula IICC (homozygous) or IiCC (heterozygous). These are dominant whites in contrast to a second type, the recessive whites, which lack the colour factor, and have the formula iicc. A dominant heterozygous white if crossed with a similar form will give descendants, IICC homozygous dominant white, IiCC heterozygous dominant white, and iiCC homozygous coloured. The homozygous white and coloured forms will breed true, while the heterozygous white will continue to throw white and coloured. 
D. Departures from the numerical expectation in the progeny of two helerozygotes. After the crossing of two heterozygotes the offspring consists of pure dominants, impure dominants, and recessives, in the proportion of $1,2,1$. But it may prove to be the case that one of the pure forms is either absolutely or relatively non-viable, and in that event we get departure from the numerical expectation. If the non-viability is merely relative, the departure will be apparent only if the counting is delayed, and if during the delay the weaker forms are exposed to the competition of the stronger.

E. Mendelian segregation of unit characters, manifesting itself as variation in the offspring of heterozygous forms, may take place in somatic or non-sexual cell division as well as in gametic division. (Bateson, 1913, p. 272.) This explains the phenomenon of bud variation in plants. The classical example of this phenomenon is the appearance of nectarines on peach trees. (Darwin, 1868, r. p. 340.) The peach is heterozygous for the character of hoariness in the fruit, with a formula $\mathrm{Hh}$ (where $\mathrm{H}$ is the dominant character for hoary, and $\mathrm{h}$ the recessive for glabrous fruit), and the nectarine is pure recessive glabrous, $h$ h. (Bateson, 1913, p. 272.) Segregation of these characters in the peach may take place at any growing point of the plant body. If it takes place in the bud, the entire branch growing from the bud bears nectarines; if it takes place at the point of origin of the fruit, one fruit is nectarine; if in the substance of the fruit, one segment is nectarine and the remainder peach.

Many additional examples of this phenomenon may be found in Animals and Plants under Domestication, of which the following may be quoted as it shows that such variation may occur with frequency and regularity. "Grapes. Count Odart describes a variety which often bears on the same stalk small round and large oblong berries; though the shape of the berry is generally a fixed character." (Darwin, 1868, I. p. 375.)

F. The stimulus determining variation. (1) Mendelian Variation. In gametic division segregation occurs at the reducing divisions with the reduction of the chromosomes. Little is known as to what stimulus determines segregation in somatic division, but nectarines appear most frequently on very old peach trees (Darwin, 1868, I. p. 341), and senescence, therefore, predisposes to if it does not cause segregation.

(2) Mutation of De Vries. Little is known of this matter also, but profound changes of surroundings are followed by outbreaks of mutation in flowering plants, for example in the chrysanthemum when first introduced into Europe from China. (Darwin, 1868, I. p. 379.)

\section{Facts observed in the group paracolon mutabile colon and THEIR INTERPRETATION.}

We shall now describe the facts observed in the group paracolon mutabile colon, and enquire how they conform to the principles contained in the above subsections A to F.

The subject may appropriately be introduced by a quotation from Darwin. 
"He who wishes to judge how far the conditions of life directly cause each particular variation ought to reflect well on the cases immediately to be given." (1868, I. p. 374.)

\section{Variation in sugar reactions.}

A. (1) True Mendelian variation in mutabile. Mutabile when grown on solid media containing lactose (Neisser, 1906; Massini, 1907; Baerthlein, 1911 and 1912), saccharose (Burri and Düggeli, 1909), or dulcite, with neutral red as an indicator, forms white colonies with colourless papillae which turn red if the particular strain varies to the sugar employed. The reddening commences in the centre of the papilla. If subcultures are made from the red papillae on media containing the same sugar, red and white colonies appear. The red colonies breed true, and never throw white descendants on the same sugar, even after prolonged subculture on media not containing it. They may, however, continue to form papillae from which variations in other characters may arise. The white daughter colonies continue to form red papillae, which again give white and red colonies in subculture ${ }^{1}$. If a strain which varies e.g. to lactose is grown on a medium not containing lactose, the papillae formed do not give descendants varying to lactose, i.e. the stimulus of the homologous sugar is required to produce the corresponding variation. Similarly a strain varying to saccharose or dulcite will accomplish this variation only on saccharose or dulcite. (Henderson Smith, 1913; Dobell, 1913.)

Variation to sugars also occurs in fluid media, for example, if a mutabile to lactose is grown in lactose peptone water the culture remains alkaline for one to seven days and then suddenly acid and gas are formed.

The facts regarding the variation of mutabile to sugars are covered if we regard this form as a heterozygous dominant white with a formula IiFF, where $\mathbf{F}$ represents the factor for the fermentation of the particular sugar (lactose, saccharose, or dulcite) to which the strain is variable, I a factor for the inhibition of F, and $i$ the absence of I. Such a form will persistently throw white non-lactose fermenting, and red lactose fermenting descendants. The formula of the latter will be iiFF, since they breed true. This derived form is indistinguishable from native colon. The white descendants should consist of the parental form IiFF, and of the homozygous dominant IIFF. We have seen that the parental form reappears, but the pure dominant has not been observed among the descendants of the papillae of mutabile. It is, however, found in nature as paracolon in close association with the other two forms. For evidence as to the formula of paracolon see the next subsection; for the explanation of its non-appearance in vitro see below, subsection D.

A. (2) Mutation in sugar reactions of paracolon. This is an uncommon phenomenon. Out of at least twenty-five strains kept under close observation

1 György (1921) states that these red colonies do not form papillae. This is incorrect. Baerthlein (1911-2) states that they throw atavistic regressions to white. I have never observed such regression in pure red strains. For the probable explanation of Baerthlein's observations see below, subsection B. Dominance. 
for not less than a year, we have found mutation to occur in three strains only, 512,1678 , and 3674 , and only on two occasions in each strain. In 512 mutation occurred in the second subculture after isolation from the bowel, in lactose peptone water and in litmus milk simultaneously. In 1678 and 3674 it occurred after culture continued over fourteen months and six months respectively. 1678 varied by the formation of papillae on a lactose MacConkey plate one month old. 3674 formed mutants in bouillon and in lactose peptone water. Except on these six occasions none of the three races ever varied to lactose, although they were frequently tested both on liquid and solid media. On the latter they formed papillae freely but these remained white and gave only white daughter colonies. They were in fact pure paracolon to lactose, although 3674 was variable to saccharose, and 1678 to dulcite.

The mutant daughter strain in 512 and 1678 proved to be a mutabile varying to lactose, in 3674 a colon. The daughter in 512 and 3674 also fermented saccharose, a sugar which the parent 512 never fermented, and the parent 3674 only after papilla formation.

In the three parent strains, therefore, we are dealing with bacteria which are permanently non-lactose fermenting, but which under the stimulus of recent isolation or senescence produce on rare occasions daughter strains with a Mendelian composition IiFF or iiFF. These facts at once suggest that the paracolon parent has a composition IIFF, and that the daughter strains arise by the dropping out of one unit character I in 512 and 1678, and of two such in 3674 , in other words by mutation of De Vries.

This interpretation is confirmed by a further observation concerning 3674 . Shortly before the appearance of the mutant described above (subrace 3305), it was noticed that colonies of the original 3674 on lactose plates were gradually becoming red about the ninth day. This change was not due to the formation of red papillae and was not heritable. Subcultures from the red colonies remained white until the ninth day, and then again gradually became red. The most satisfactory explanation of this observation appears to be, that in a race with a formula IIFF the inhibitory II had, through senility, become partly ineffective. The case is exactly similar to that of hornless breeds of cattle, "some of which acquire as they grow old small horns." (Darwin, 1868, II. p. 54.) The hornless breeds are those in which the development of the horns is prevented by an inhibitory factor. (Punnett, 1919, p. 79.)

It may be suggested that the appearance of lactose fermenters in nonlactose fermenting cultures was due not to mutation but to contamination. That this was not the case is shown by the fact that in 3674 the lactose fermenter 3305 was agglutinated to titre limit by antiserum prepared against the non-lactose fermenting parent. In 512 this coagglutination took place occasionally although not constantly, while 1678 has not been tested in this respect, but the origin of the daughter from papillae on the body of the parent allowed of little doubt as to her legitimacy.

Neisser and Massini consider the variation in mutabile to be a mutation 
in the sense of De Vries. By implication, therefore, we may assume that they regard the parent form as lacking a lactose fermenting factor, which is acquired by interpolation in the red descendants ( $f$ is converted into $F$ ). Three arguments suggest themselves against this view. (1) An interpolation would hardly occur and recur with such regularity in many races as to enable us to predict that on a given day every culture would form the red variant, whereas if the formula of white is IiFF the normal process of segregation will give this regular variation. (2) If interpolation took place so regularly, the reverse variation by dropping out should occur still more regularly, F giving $f$, but we have seen that this does not occur; the red form never returns to the white, a fact fully explained by the formula iiFF for the red. (3) On Neisser and Massini's hypothesis how can we explain the difference between the variation in mutabile and paracolon? If in both the change is due to the same cause-the interpolation of a fermenting factor-why does not paracolon vary as frequently and regularly as mutabile?

I am not acquainted with any change in sugar reactions which corresponds to mutation by interpolation of a factor, but the rare reversion from "rough" to "smooth" reported by Arkwright $(1921,1924)$ may be an example of this process. Arkwright, because of these reversions, denies the irreversibility of the change from "smooth" to "rough." The doctrine of irreversibility might perhaps be more accurately stated by asserting that the change is not regularly reversible by that routine Mendelian segregation which causes it, but this does not exclude rare reversion by interpolation of the character previously lost.

B. Dominance. On lactose plates with neutral red as an indicator, mutabile to lactose, with a formula IiFF, appears in the form of white colonies. In some strains they remain dead white until the plate dries up, in other words the dominance of $I$ over $i$ is complete. In other strains, however, after a few days, the colonies acquire a faint red tinge in the centre, which may deepen and spread over the whole colony. The dominance in this case is not complete, and the colour of the heterozygote is intermediate between that of the pure white dominant paracolon, IIFF, and the recessive red colon, iiFF. Even in strains descended from a common parent one may remain pure white, while another becomes pink. Nevertheless the pink of this heterozygote with incomplete dominance is never so vivid as the red of the pure recessive, and the two forms growing on the same plate can readily be distinguished. I believe, however, that this incomplete dominance may account for some reports of frequent reversal of the variation white to red. Baerthlein (1911 and 1912) has reported such cases. It is probable that the form which he took for a red was really an elderly white with incomplete dominance. Such a form would regularly throw daughters which would be white for the first few days of their life at least.

Toeniessen (1921) explains the changes observed by him in the development of the capsule in the pneumobacillus of Friedländer by this variation in dominance. The changes were heritable but could be reversed without difficulty, 
and all degrees from a fully developed capsule to complete nakedness were observed.

D. Departure from the numerical expectation in the offspring of two heterozygotes. Mutabile as a heterozygote of formula IiFF should throw offspring in the proportion of one dominant white, IIFF, two heterozygous whites, IiFF, and one pure recessive red, iiFF. It is obvious that no attempt can be made to count the actual descendants, but we should expect to find the three types among them. In this, however, we are disappointed, since only the two latter appear. To discover some explanation of this irregularity, mixed cultures of paracolon, of the white form of mutabile, and of the red or derived colon were made, in lactose peptone water and on lactose MacConkey plates. It was found that paracolon would survive in competition with colon, but seldom with the white form of mutabile. The derived colon on the other hand, and the white mutabile, came up quite readily together. The non-appearance of the dominant white is, therefore, probably explained by its failure to survive in competition with its heterozygous parent.

E. Does the variation from white to red in mutabile take place in gametic or in somatic (i.e. non-sexual) cell division? Does it correspond with seminal or bud variation in higher plants? It occurs at one point of the colony only, namely the papilla (although the entire colony is exposed to the same stimulus which determines segregation in the papilla-see below, subsection F). Therefore the cell divisions in the papilla differ from those occurring in the rest of the colony, and since in them segregation of unit characters occurs so constantly as to manifest its presence by variation whenever the parent is heterozygous, we are forced to the conclusion that the papillary divisions in bacteria correspond in some way with the reducing or gametic divisions of higher forms. Whether the papillary cell division is or is not followed by conjugation may remain an open question for the present. Failing conjugation some form of duplication of unit characters corresponding to a theoretically pure parthenogenesis might be postulated; thus after a gametic division of IiFF to IF and iF, iF may duplicate to iiFF the red variety, while the undivided IiFF continues as the white variety.

Although it is not necessary for our thesis to prove that the papilla is a sexual organ, the following considerations bear upon the subject. The papilla in its macroscopic appearance strongly recalls the fructifications of some of the higher hyphomycetes-e.g. the sporocarp of Eurotium. Almquist (1923-4) has recently claimed to have demonstrated sexual conjugation in $B$. typhosus and $B$. dysenteriae. To ascertain whether conjugation could be proved between paracolon and colon, I performed the following experiments. Culture tubes were inoculated with strains of paracolon and colon which were forming papillae actively, and after incubation the mixture was plated in the hope that mutabile might have been formed by the crossing of the two original strains. Mutabile did not appear, but the conditions may not have been favourable, and the experiment deserves repetition. 
Consideration of the variation smooth to rough will illuminate the distinction which we are trying to make between the papillary variation, and variation in somatic division. On plating any bacillus on solid media it will often be found that colonies of two types appear, firstly smooth, circular, convex, opaque, and secondly rough, irregular in shape with crenated margin, flat and translucent. The rough type breeds true, the smooth type gives both smooth and rough. Smooth is, therefore, a heterozygous dominant, Sr, while rough is a homozygous recessive, rr. Now rough does not arise from a papilla on a smooth colony, but makes its appearance at any point of the growing margin, and after further growth the colony presents a striking appearance of alternating sectors of rough and smooth. This variation, therefore, takes place in somatic division, in a manner exactly comparable to the peach, nectarine, or oval and round grape variation. Comparing this variation with the papillary, it is difficult to resist the conclusion that the papilla is sexual.

Papillae are structures of obviously specialised character, sharply defined from the remainder of the colony, of definite shape, and in their primitive form of characteristically small size. If a favourable variation occurs in the interior of the papilla, the new race thus formed grows upwards and outwards as a victorious daughter colony. This secondary development should not be confounded with the true papilla. If no favourable variation arises, as in the case of paracolon, the papilla remains minute, compact and colourless. György has regarded the papilla as a daughter colony, that is, as the outcropping of a modified race, which, owing to favourable variation, overgrows the parent race. Such an interpretation offers no explanation of those stationary, non-developing papillae in which no favourable variation arises.

F. Variation determined by an external stimulus. Variation in mutabile differs from Mendelian variation in higher forms in the fact that not only is a special form of cell division (the papillary or gametic) required, but a definite external stimulus, lactose, saccharose, or dulcite must also be present. Papillae formed in the absence of the sugar to which the form varies do not give the red variety. It is obvious that bacteria are in much more intimate contact with their surroundings than are the sex cells of higher forms, and it is, therefore, not unreasonable to expect such a deviation from the laws governing segregation in the latter.

\section{Variation by capsulation.}

Variation from the naked to the capsulated condition was observed in race FMG. 512, which, commencing as paracolon, gave subraces which in succession acquired the power of fermenting saccharose, lactose, and dulcite. It had thus arrived at a condition corresponding with $B$. coli communior (see Table, subrace 2169). At this point it was grown on a plate of dulcite MacConkey's medium so thinly sown that only two colonies appeared, and after fifteen days, when the plate was becoming somewhat dry, a large gelatinous papilla appeared on each colony. On subculturing from these papillae on dulcite, gelatinous colonies of capsulated bacilli appeared. On purification this subrace was found 
to give, on dulcite, gelatinous colonies which breed true, 3351, and nongelatinous colonies which formed gelatinous papillae, 3353. From the gelatinous papillae again came daughter colonies gelatinous and non-gelatinous. We are here clearly dealing with a form heterozygous for the capsulated (gelatinous) condition, in which the non-capsulated is dominant to the capsulated. This case is exactly similar to that of mutabile, where the non-lactose fermenting state is dominant to the lactose fermenting, and as we represent the latter by the formula IiFF, we should represent the former by IiCC, where $\mathrm{C}$ is the factor for capsulation. The permanently non-capsulated parent, subrace 2169, must then have a formula IICC (as paracolon 512 is IIFF) and the process by which it gave rise to $\mathrm{IiCC}$ was again the rare mutation of De Vries by the dropping out of one I factor. This supposition is confirmed by the fact that further cultures of 2169 did not again yield capsulated descendants. The change from 3353 to 3351 , on the contrary, recurs regularly after every papilla formation, since IiCC segregates at gametic papillary division, and gives descendants $\mathrm{IiCC}$ and $\mathrm{iiCC}$ - the latter being the pure gelatinous race 3351 .

A further complication is introduced by transferring the gelatinous race 3351 from dulcite to lactose, since it proves non-gelatinous on the latter. But if 3353 is grown on lactose it forms papillae which give descendants, 3196, capable of capsulating both on lactose and dulcite. 3196 can also capsulate on glucose but not on saccharose.

These observations reveal how extraordinarily individual and narrowly circumscribed each unit character may be. Nevertheless, each is exactly inherited even after growth on indifferent media on which the unit character necessarily lies dormant.

At this point it appears desirable to refer to the work of Avery and Heidelberger (1925) on the capsules of pneumococcus. These authors find that the capsule of each of the three types of this organism is composed of a different polysaccharid, and that it is upon this difference that the specificity of response in the antiserum to each type is dependent. Toeniessen (1920) states that the capsule of $B$. pneumoniae Friedländer is composed of galactane, a polysaccharid compound of galactose.

The capsulated types of our race F.M.G. 512 are agglutinated by an antiserum to the non-capsulated parent, but only in much lower dilution than the titre.

Conclusion as to the systematic position of paracolon mutabile colon and pneumobacillus of Friedländer. To sum up the facts elicited by the above enquiry as they bear on the systematic position of the bacteria investigated, we find that the entire series of paracolon, mutabile, colon, and pneumobacillus of Friedländer are Mendelian variants of one species. In paracolon-mutabilecolon, mutabile is the central heterozygote, paracolon the pure dominant, and colon the pure recessive.

The correct designation of this species appears to be Bacillus neapolitanus Emmerich, 1885. 
. Yot showing routine Mendelian variation in sugar reactions. Not fermenting lactose.

Subgroup Paracolon.

Sect. (1) F.J.L. 1967. F.M.G. 1643, 2133. J.S. 1969

(2) F.M.G. 113, 510. F.O. 349 F.M.G. 512

\begin{tabular}{|c|c|c|}
\hline 01 & & \\
\hline , & subrace & 2638 \\
\hline , & , & 2817 \\
\hline , & ", & 2473 \\
\hline " & , & 2169 \\
\hline ", & , & 3353 \\
\hline ," & $"$ & 3351 \\
\hline "2003 & ", & 310 \\
\hline
\end{tabular}

(4) M.A.T.

I. Showing routine Mendelian variation in sugar reactions.

Subgroup Mutabile (a) Not fermenting lactose

A. Varying to lactose alone

(1) T.A.B. 1552

(2) J.B."139. F.S. 249

(4) E.B. 805 " subraces $R / L$ F.M.G. 2669

" subrace $\mathrm{R} / \mathrm{L}$

B. Varying to saccharose alone

(1) F.M.G. 2356

F.S. 3674 subrace R/S

" subrace 1900

" $\quad$ " $\quad 3305$

C. Varying to dulcite alone

(1) R.W. 1842, F.J.L. 1731

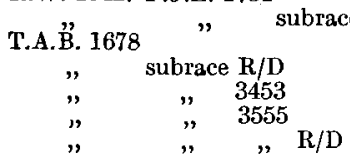

D. Varying to lactose and dulcite

(3) J.R. 506, 508

$$
\begin{array}{rrr}
, & \text { subrace } & 1899 \\
, & " & 1887 \\
" & " & 1888
\end{array}
$$

Subgroup Mutabile (b) Fermenting lactose, i.e. variable colon

\section{(4) Mon 4284}

" subrace

$A G \quad A G \quad A G \quad A G \quad A G \quad-\quad A C$

$\overrightarrow{A G} \quad A G \quad A G \quad A G-A K$

$\begin{array}{lllllll}A G & A G & A G & A G & A G & - & A \\ A G & A G & A G & A G & A G & A G & A C\end{array}$

- AG AG AG AG AG AK
- $\overline{A G}$ AG $A G$ AG $A G=A$

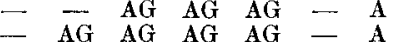

$A G$ AG AG AG AG $-A C$

$A G$ AG $A G$ AG $A G$ AG AC

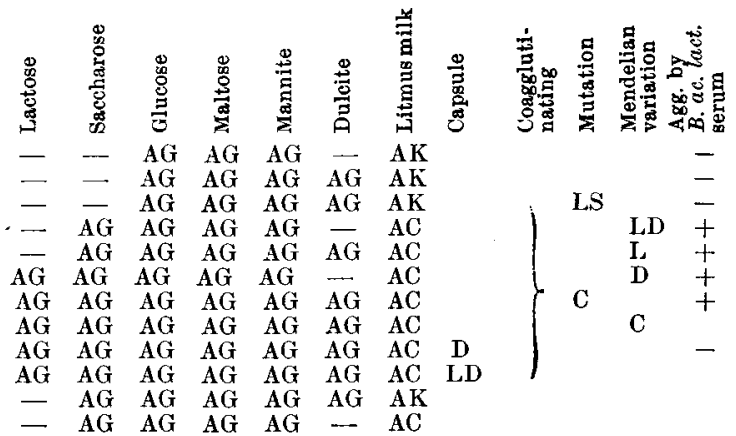

$\mathrm{D}$

L D -

$\begin{array}{ll}\mathrm{LD} & - \\ \mathrm{D} & -\end{array}$

$\begin{array}{ll}\text { LD } & + \\ \mathbf{D} & +\end{array}$

II. Not showing routine Mendelian variation in sugar reactions.

Fermenting lactose

Subgroup Colon

(1) B. acidi lactici

(2) B. coli communis

(3) B. coli communior

$B$. pneumoniae Friedländer

(4) B. lactis aerogenes

4151

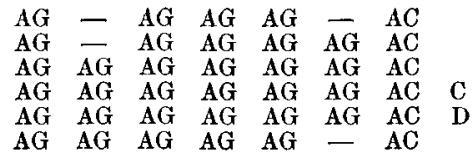

C $\quad \frac{+}{-}$ 


\section{DeduCtions BEARING ON THE THEORY OF ACQUIRED VIRULENCE.}

The phenomena attending the acquisition by mutabile of the power of fermenting a sugar, and the parallel capsulation in colon, and rough variation, are of great importance from the point of view of pathological theory. We find that the white form of mutabile is restrained from attacking the sugar by an inhibitory factor, that by exposure to the sugar it forms a Mendelian variant lacking this inhibitory factor. It has in fact become virulent to the sugar. This development of virulence to a carbohydrate is clearly a simple matter compared with the development of virulence to a living organism. We may enquire whether there are any facts which would support such a parallel, and whether the development of pathological virulence is due to Mendelian variation.

(1) Hobart N. Reimann by growing a virulent strain of pneumococcus in bouillon to which serum had been added, observed the appearance of a variant. This variant was non-virulent to guinea-pigs, it was also non-capsulated and formed rough colonies, while the parent strain was virulent, capsulated and smooth. Pure cultures of the variant never on any culture medium returned to the parental form, and no intermediate was observed. We are here clearly dealing with Mendelian variation from virulent to non-virulent.

(2) Toeniessen (1921), for the pneumobacillus of Friedländer, found that a capsulated strain was virulent to mice, a strain which had lost its capsule in vitro was non-virulent, and virulence decreased parallel to the loss of the capsule. On restoring virulence by animal passage the capsule was found to be restored also.

We have seen that some at least of these reversible variations of Toeniessen are due to changes in dominance of the I factor. He claims, however, that other and more rare types are due to mutation. From our present point of view the parallelism between capsulation and virulence is the important fact.

(3) Baerthlein (1918), working with Friedländer's bacillus from a case of intestinal catarrh, isolated three variants, of which only one formed gelatinous colonies and was capsulated. This variant alone was virulent to mice. Agar plates from the heart blood gave both gelatinous and non-gelatinous colonies.

He also found that in $B$. diphtheriae virulent strains can be separated as variants from avirulent parents.

(4) MacGowan and Chung Yik Wang (1915-16) found that an organism of the haemorrhagic septicaemia group, the virulence of which had been exalted by animal passage, had been concurrently endowed with greater biological activity in vitro, that it produced acid and gas from sugars which it previously had not attacked or from which it had produced acid only. The relatively nonvirulent parent gave acid only, in lactose, glucose, maltose, and mannite, and did not ferment saccharose, while the virulent descendant gave acid and gas in all five sugars. Retrogression from virulent to non-virulent was extremely slow, little change taking place in three months. Antisera against the parent 
and the derived form did not cross agglutinate, an indication of the profundity of the change which had taken place.

(5) Georg Bernhardt (1915) states that meningococci form papillae in the same manner as colon. The organism grown from the papillae is more hardy in culture than the parent.

(6) Oswald T. Avery and Michael Heidelberger (1925) find that the virulence and specificity to antisera of the various types of pneumococcus are in direct relationship with the development of the capsule. The specificity is dependent on the inclusion of a different sugar in the capsule of each type. The specific carbohydrate isolated from the capsule of each type is precipitated in a dilution of $1 / 5,000,000$ by the antiserum of its own type only.

Rebecca Lancefield (1925) obtains corresponding serological results with the carbohydrate extracts of Streptococcus viridans.

We find, therefore, that in a number of different species of bacteria there is an emphatic parallelism between pathological virulence on the one hand, and the degree of development of the capsule, and of the power of attacking sugars on the other. We have found that the acquisition of a capsule and of the power to attack sugars follows Mendel's laws. It appears permissible, therefore, to assume that the acquisition of pathological virulence will be subject to the same principles as the acquisition of virulence to sugars. This latter is determined by the presentation to the organism of the sugar to be attacked, and by the response of the organism by the loss of a factor (a Mendelian unit character) which inhibited it from attacking the sugar. The organism is heterozygous for this factor, and its power of immediate response is dependent on this heterozygous condition.

Acquired pathological virulence should, therefore, be due to the presentation to a commensal organism of an antigenic molecule of the host in a free condition, and the commensal should be heterozygous for an inhibitory factor preventing it from attacking the host. A microbe incapable of becoming parasitic will differ from one so capable by being homozygous for the inhibitory factor.

The action of the precipitating cause of a disease, such as chill or excessive exertion, would be the liberation of this antigenic molecule, and thus we can explain the onset of $e . g$. pneumonia, enteritis, or the common cold.

The existence of such inhibitory factors has long ago been anticipated from general biological principles. A lowly organism in the earliest stages of evolution would be adapted to attack any other body on which it could feed. But preservation of the race would soon develop an inhibition to attacks on members of its own race, and of races helpful to it. This inhibition would become more imperatively necessary on the assumption of the multicellular state, and the development of commensalism. In fact a widespread system of inhibition must exist on a low physiological plane, as it exists on the psychological plane in the control of the primitive instincts. On both levels the inhibitory powers are developed by social interplay. 
It is possible to construct a classification of various forms of acquired virulence in terms of biological theory as follows:

A. Arising by mutation of De Vries, the dropping out or interpolation of new factors. This would be of rare occurrence and would be rarely reversible. Such mutation would explain the appearance of a new disease such as encephalitis lethargica, and possibly the recurrence and recession of such a disease as epidemic influenza.

B. By Mendelian variation. Of regular occurrence when the appropriate stimulus is presented, and non-reversible, e.g. pneumonia, wound infection, peritonitis.

C. By change in dominance of the inhibitory factor-Valenzwechsel der Erbfaktoren of Toeniessen. Precipitating cause doubtful; reversible.

D. By modification. The variation to virulence continues only during the continuance of the accessory external influence which provoked it, and the virulence is not heritable. Diseases in which an accessory chemical factor is required, such as the Peyton Rous sarcoma.

\section{Clinical significance of an Increase of paracolon or MUTABILE IN THE BOWEL.}

During the past four years systematic observations have been carried out in the laboratory of this hospital as to the proportion of non-lactose fermenting to lactose fermenting coliforms in the faeces, and a considerable number of persons have been examined in this respect. We have confined our attention strictly to the coliform group, and have endeavoured to ascertain whether there is any constant relation between variation in health and variation in the relative number of paracolon, mutabile, and colon in the faeces.

Previous literature. Morgan and Ledingham (1909) state that in diarrhoea the non-lactose fermenting groups increase. This increase may be so marked as to lead to the complete exclusion of the lactose fermenters. They consider, however, that the paracolon group is of little pathological importance.

György (1921) regards the paracolon group as important from its presence in the faeces in diarrhoea. It is aetiological in diarrhoea of calves and in cholera infantum. He, however, includes paratyphoid $\mathrm{C}$ in the paracolon group.

The results obtained in this hospital may be briefly summarised as follows.

A. Presence or absence of paracolon and mutabile in the faeces of a series of 253 unselected cases.

The patients examined are divided into three categories:

(1) Acutely ill: fresh admissions or acute relapses: cases under the clinical headings of acute confusional insanity, recent manic depressive insanity, acute alcoholism, primary dementia, and general paralysis of the insane in the acute phase. It may be assumed that these patients were all suffering from gastrointestinal disturbance with accompanying disturbance of general bodily health. 
(2) In subacute or chronic ill health: including epileptics, general paralytics, chronic primary dements, and seniles.

(3) In good bodily health.

The following percentages of the individuals in the three classes showed paracolon or mutabile in their faeces. Class (1) 31.8 per cent. out of 113 cases gave paracolon or mutabile or both : paracolon present in 14 per cent., mutabile in 24 per cent. Class (2) $25 \cdot 3$ per cent. out of 86 cases gave paracolon or mutabile or both: paracolon present in 12.7 per cent., mutabile in 15 per cent. Class (3) 3.7 per cent. out of 54 cases gave paracolon or mutabile or both: paracolon present in 1.85 per cent., mutabile in 1.85 per cent.

B. Presence or absence of paracolon and mutabile in the faeces of a series of thirty-eight cases of acute manic depressive and confusional insanity.

$\begin{array}{lllll}\text { Cases with paracolon } & \ldots & \ldots & \ldots & 9\end{array}$

Cases with mutabile $\quad \ldots \quad \ldots \quad \ldots \quad 12$

Cases with paracolon or mutabile or both 19

Cases without paracolon or mutabile $\quad \ldots \quad 19$

Out of the above nineteen cases showing paracolon or mutabile or both, in fifteen the proportion of colon mutabile to the total coliforms was above 20 per cent., varying from 20 to 100 per cent.

C. Variation in the proportion of paracolon mutabile to total coliforms in the course of an illness.

Out of the above series of manic depressive and confusional cases, eight recurrent cases were selected for special study, faeces being plated at regular intervals, and the proportion of non-lactose fermenting coliform colonies charted in comparison with the development and progress of the disease. It was found that in the intervals of relative health, non-lactose fermenters were absent, or present in only small numbers. When an attack began they appeared in proportion varying from 20 to 100 per cent. They increased with increase of symptoms and diminished with abatement. While this rule was general it was not absolute - e.g. in one patient who was followed through five attacks in the course of four years, the rule held for the first three attacks, but in the next two paracolon or mutabile were only found on two occasions.

We find, therefore, that there is a close but by no means absolute connection between paracolon mutabile and many different forms of ill-health. Paracolon mutabile bacteria are obviously not the primary cause of the disease. Their appearance is symptomatic rather than aetiological, although they may have some power for the development of secondary ills. The disordered condition of the intestine may determine the change in the flora, (1) by encourag. ing an overgrowth of paracolon mutabile previously present in small numbers, or (2) by promoting an active mutation from the colon to the paracolom 
mutabile type by the spontaneous appearance of factors inhibiting sugar fermentation. This mutation will doubtless prove to be a linked variation, linked with some variation to protein metabolism, which is directly caused by the stimulus of altered protein environment in the bowel. It is necessary to realise that our study of the variation in the species $B$. neapolitanus Emmerich has been entirely one-sided, that is, from the point of view of carbohydrate metabolism. It is probable that another series of variations could be obtained from the point of view of protein metabolism; that paracolon which is relatively inhibited to carbohydrates will prove relatively active to proteins, that colon which is relatively active to carbohydrates will prove relatively inhibited to proteins, while mutabile will hold a middle place from both aspects.

The return of the intestinal flora from paracolon mutabile to colon, accompanying the transition from sickness to health; will doubtless be due to a change in the carbohydrate environment in the bowel-such as an increase in assimilable sugar.

In conclusion I wish to express my indebtedness to Mr W. F. Gifford, senior assistant in the laboratory of the County Mental Hospital, Cheddleton, for his generous help in this investigation during the past two years.

\section{LITERATURE.}

Armouist (1923). Zeitschr.f. Hyg. c. 15.

- (1924). J. Infect. Disease, xxxv. 341.

ARKwright (1921). J. Path. and Bact. xxiv. 36.

ARKWRTAHT and GoYLe (1924). Brit. J. Exper. Path. v. 104.

AVERy and HeIdelberger (1925). J. Exper. Med. xIII. 367.

Baerthldein (1911). Centralbl. Bakt. Abt. I. Ref. L. Beiheft, p. 128.

- (1912). Ibid. Orig. LXVI. 21.

(1918). Ibid. LXXXr. 369.

Bateson (1913). Mendel's Principles.

BernhaRdT, G. (1915). Zeitschr. f. Hyg. LXXIX. 179.

Burck, A. (1908). Arch. f. Hyg. Lx. 235. (Summary in Bull. Inst. Pasteur, 1908, p. 639.)

Burri (1910). Centralbl. Bakt. Abt. r. Orig. xxvIII. 321.

BURRI and DÜGGelI (1909). Ibid. Abt. I. Orig. XLIX. 143.

DarwiN (1868). Animals and Plants under Domestication.

Dobell $(1912,1913)$. J. Genetics, ㅍ. 285, 325.

GrLBerT and LION (1893). Semaine Méd.

Gxöray (1921). Centralbl. Bakt. Abt. I. Orig. Lxxxiv. 321.

Herrold and Colver (1919). J. Infect. Disease, xxx. 114.

LANCEFIELD (1925). J. Exper. Med. XLIL. 337, 397.

MagGowan and Chung Yik Wang (1915-1916). J. Path. and Bact. xx. 21.

MaCkIE (1913). Ibid. XVIII. 137.

Massini (1907). Arch. f. Hyg. Lxr. 250. (Summary in Bull. Inst. Pasteur, 1907, p. 791.)

Morgan (1906). Brit. Med. Journ. I. 908. (1907). Ibid. Ir. 16.

Morgan and Ledingham (1909). Proc. R. Soc. Med. Epidemiol. Sect. p. 133.

Müller, Reiner (1909). Centralbl. Bakt. Abt. I. Ref. xurr. Beiheft, p. 57.

- (1911). Ibid. Orig. Lvirr. 97. 
Neisser, J. M. (1906). Ibid. Abt. I. Ref. xxxvir. Beiheft, p. 98.

Pentold (1911). J. Hygiene, xi. 30.

(1911). Proc. R. Soc. Med. Iv. Pathol. Sect. p. 97.

Punnett (1919). Mendelism.

ReIMANN (1925). J. Exper. Med. LXI. 589.

Savage and Bruce (1925). Med. Res. Council, xcI.

SCHÜTze (1922). J. Hygiene, xx. 330.

Smith, Henderson (1913). Centralbl. Balt. Abt. I. Orig. Lxviri. 151.

Toeniessen, E. (1920). Ibid. Lxxxv. 225.

— (1921). Ibid. Lxxxvi. 353.

(MS. received for publication 21. IV. 1926.-Ed.) 